\title{
BMJ Open Patients' views on the causes of their polymyalgia rheumatica: a content analysis of data from the PMR Cohort Study
}

\author{
Maatla Tshimologo, ${ }^{1}$ Benjamin Saunders, ${ }^{1}$ Sara Muller, ${ }^{1}$ Christian D Mallen, ${ }^{1}$ \\ Samantha L Hider ${ }^{1,2}$
}

To cite: Tshimologo $\mathrm{M}$, Saunders B, Muller S, et al. Patients' views on the causes of their polymyalgia rheumatica: a content analysis of data from the PMR Cohort Study. BMJ Open 2017;7:e014301. doi:10.1136/bmjopen-2016014301

- Prepublication history for this paper is available online. To view these files please visit the journal online (http://dx.doi.org/10.1136/ bmjopen-2016-014301).

Received 15 September 2016 Revised 7 December 2016 Accepted 14 December 2016

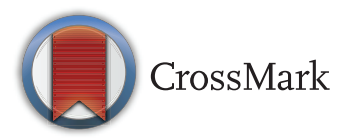

\footnotetext{
${ }^{1}$ Research Institute for Primary Care \& Health Sciences, Keele University, Keele, UK

2Department of

Rheumatology, Haywood Hospital, Keele, UK
}

Correspondence to Dr Benjamin Saunders; b.saunders@keele.ac.uk

\section{ABSTRACT}

Objective: To explore primary care polymyalgia rheumatica (PMR) patient beliefs about the causes of their PMR.

Design: Qualitative content analysis was conducted on patients' written responses to the question of what they thought had caused their PMR. All data were coded and emergent categories of causal beliefs identified. Setting: Community patients receiving primary care at general practitioner (GP) practices across England.

Participants: Participants were recruited from a primary care PMR inception cohort $(n=654)$. Between June 2012 and June 2014 GPs referred 739 people with a new PMR diagnosis in the past 3 years into the study. Patients were mailed a baseline self-completion questionnaire, which included the question, 'What do you think caused your PMR?'. Responses to this question form the data set for the present study. Results: 296 (45\%) patients gave a possible cause for their PMR, while $276(42 \%)$ respondents wrote 'no idea'. Common attributions include ageing $(45,18 \%)$, medication $(18,5 \%)$ and personal stress $(53,14 \%)$. 24 respondents $(6 \%)$ thought their PMR was as a result of another medical condition.

Conclusions: This is the first study to examine causation beliefs in PMR, identifying a number of possible causes such as ageing, stress and as a complication of other medical problems. Understanding these patient beliefs may impact on treatment adherence and patient outcome.

\section{INTRODUCTION}

Polymyalgia rheumatica (PMR) is a common inflammatory disease of older adults, which is more prevalent in females than males and is predominantly found in the Caucasian community. ${ }^{1}$ PMR classically presents with a sudden onset of bilateral myalgia/pain in the shoulder and hip girdles as well as systemic features such as weight loss, nausea and fever. $^{2}$ The aetiopathology of PMR is currently not well understood, ${ }^{2}$ although studies

\section{Strengths and limitations of this study}

- A strength of this study is the large sample size which is representative of the primary care polymyalgia rheumatica (PMR) population.

- Respondents in this study had been very recently diagnosed with PMR prior to questionnaire completion, therefore minimising recall bias.

- The content analysis approach used allowed for an unobtrusive and non-reactive way to study patients' opinions.

- Analysis of short written responses did not allow for consideration of broader contextual factors related to patients' beliefs, and therefore future in-depth qualitative research could give further insights.

suggest genetic factors including human leucocyte antigen shared epitope and polymorphisms in proinflammatory cytokines such as tumour necrosis factor- $\alpha$ and interleukin-6 cluster genes may be implicated. ${ }^{2}$ Infections and vaccinations (eg, influenza) have also been linked to slightly higher risk of developing PMR, although to date, these studies are inconclusive. ${ }^{2-6}$

Patient beliefs about the causation of illness can have a significant effect on the attitude patients have about their illness, the stigma they attach to it and may impact on their health-seeking behaviour and attitudes towards treatment. ${ }^{7}$ Qualitative studies of patients with rheumatoid arthritis (RA) suggest that patients believed their RA was triggered by a psychological factor or life event, whereas patients with spondyloarthritis (SpA) felt that their disease was as a result of a genetic predisposition. ${ }^{8}$ These studies have helped clinicians gain better understanding of the views of patients with $\mathrm{RA}$ and $\mathrm{SpA}$ about the origins of their condition which may lead to improvements in care by promoting dialogue on the psychological impact of 
disease, and advice on suitable coping mechanisms and understanding expectations of treatment. ${ }^{8}$ To date, there are limited data on patients' beliefs about PMR or its cause. This study aims to explore primary care PMR patient beliefs about the causes of their PMR.

\section{METHODS}

\section{Patient cohort}

Respondents in this study were recruited from a primary care PMR inception cohort (see ref. 9). Briefly, patients were recruited from 382 general practitioner (GP) practices across England. Between June 2012 and June 2014 GPs referred 739 people with a new PMR diagnosis in the past 3 years into the study. PMR diagnosis was made by GPs, who were provided with British Society for Rheumatology (BSR) guidelines on diagnosing PMR. Patients were mailed a baseline self-completion questionnaire. This included questions relating to PMR symptoms and severity, treatment, functionality, general health, lifestyle habits and sociodemographic factors, and included an open question, 'What do you think caused your PMR?'. Responses from this question provided the data for this study. All patients provided written informed consent.

\section{Data analysis}

All of the patients' written responses to the question about the causes of their PMR were first separated from the original questionnaires and stored on a separate password-protected spreadsheet for analysis, in order to ensure that written responses could not be linked to information in the questionnaires that could identify patients. The responses were then fully anonymised, with any potentially identifiable information, for example, names of people or places, removed or replaced by pseudonyms.

Respondents' answers were analysed using content analysis. This is a systematic method of ascribing meaning to qualitative data in order to identify recurrent patterns. ${ }^{9}$ First, data were read repeatedly by a single author (MT) to achieve data immersion. Second, data were classified according to emergent descriptive codes that were identified. For example, the following response was coded as 'occupational strain': “...my work, climbing poles and ladders, leaning out on my belt connecting wires and services", while "... a road traffic accident as a backseat passenger in a stationary car outside my home" was coded as 'injury'. The following response was coded as 'no idea': "I simply have no idea".

Throughout the coding process, keywords were identified within the respondents' answers. This sometimes entailed picking up the main idea expressed in the sentence and making inferences as to what was being implied. For example, a response such as, "I don't know but my mum had it", indicates a hereditary cause, despite the participant not saying this explicitly. In these cases coding was more interpretive, moving beyond a face value description of the respondents' answers. An iterative, reflexive approach was adopted, whereby codes were continuously revised and refined throughout the analysis until data saturation was reached, with no new codes emerging.

The mean age and SD for the whole cohort was calculated. The number of participant responses within each preliminary code was counted. Codes that were seen to be closely related in meaning were then combined into broader categories, resulting in the identification of 11 main categories. The emergent categories were examined in relation to respondents' demographic characteristics.

A random sample of 100 responses was independently coded by another of the authors (BS) to check for intercoder reliability. Coders brought different disciplinary perspectives to the data (MT is from a clinical background, BS from a social science background), and the aim of independent coding was therefore to understand cross-disciplinary perspectives on the data and, through discussion, to come to an agreement on shared meanings and interpretations. The level of agreement between coders was therefore assessed through detailed discussion, rather than statistical calculation of levels of agreement.

The respective backgrounds of the two coders must be taken into account when interpreting the data, as their own subjectivities will inevitably have an influence on how the patients' responses are interpreted. However, this need not represent a limitation and can instead be seen to form an integral part of the analysis process. Despite their differing disciplinary perspectives, the two coders were largely found to agree on the main categories, and where any disagreement was apparent this was resolved through discussion of individual cases, which resulted in agreement on the final categories. This intercoder agreement can be seen to enhance the trustworthiness of the findings presented.

\section{RESULTS}

\section{Main findings}

Of those mailed, $654(88.5 \%)$ returned the baseline questionnaire. Of the 654 responders $408(62.3 \%)$ were female and the mean (SD) age was $72.4(9.2)$ years. The majority of the sample were retired $(n=513,79.9 \%)$, with only $77(11.9 \%)$ reporting themselves to be employed. The sample almost all identified themselves as being of white ethnicity $(n=640,98.2 \%)$. In terms of occupation, approximately a third of the sample had or previously held higher managerial, administrative or professional occupations, while $28 \%$ had intermediate occupations (eg, higher grade white collar workers) and $40 \%$ had routine occupations (semiskilled and unskilled work, eg, production, technical or operative job roles). Of the 654 respondents, $296(45 \%)$ gave a possible cause in answer to the question, 'What do you think caused your PMR?'. Two hundred and seventy-six (42\%) respondents wrote 
'no idea' and $82(13 \%)$ left the question blank. The 296 respondents who gave a possible cause were slightly younger, mean age of 71.4 (SD 9.9) years and $63 \%$ were female. The non-responders/'no idea' group had a mean age of 73.0 (SD 8.5) years and $62 \%$ of this group were female. Of those who suggested possible causes, $159(54 \%)$ respondents reported multiple factors. The multiple factors given by respondents resulted in the total number of responses given $(n=379)$ being more than the number of individuals $(n=296)$. The initial number of codes totalled 37; though some related to answers provided by only one or two respondents. Eleven broader categories were identified from this initial set of codes. Some causes did not fall under a main category and therefore stood alone; for example, falls, muscle strain, road traffic accident injuries were all grouped to make one umbrella category of 'injury'; while 'personal stress', not falling under any category and having a large number of respondents, stood alone as a main category.

The main categories of PMR causes given by respondents were: occupational strain, personal stress, age and age-related conditions, medication, extension of an existing musculoskeletal disease, linked to another nonmusculoskeletal illness, injury, genetic causes, environmental, lifestyle and lifestyle-related conditions, medical intervention complications and infection (table 1).

The three commonest causes cited were injury, personal stress, age and age-related conditions, respectively. The least common causes were environmental, consequence/complication of medical intervention and lifestyle and lifestyle-related conditions.

\section{Causal attributions and participant variables}

Respondents who attributed their PMR to age/normal wear and tear of the body had an average age of 74.5 years (range 58-91 years). This group was predominantly male (57\% vs $43 \%)$ and mostly gave single causes for their PMR, for example: "My age, worn out joints" (79-year-old woman); "I would suggest age is the major factor" (78-year-old man).

Respondents who suggested that their PMR was a result of an existing musculoskeletal condition had an average age of 70.4 years (range 48-94 years) and were predominantly female ( $76 \%$ vs $24 \%$ ). Only $21 \%$ of the individuals citing an existing musculoskeletal condition gave multiple causes. Several musculoskeletal diseases were mentioned, including the following examples: "I thought suffering for years with arthritis was the cause of PMR" (81-year-old woman); "I have no idea, although I do have Arthritis in knees, hands+think perhaps this may have been the cause" (76-year-old woman).

For respondents who attributed their PMR to personal stress, the average age was 67.2 years, and the age range was $38-89$ years. These respondents were predominantly female $(80 \%$ vs $20 \%)$. The stressful situations that were highlighted included bereavement, caring for a
Table 1 Participant reported causes of PMR and breakdown of the main categories (the main categories and their overall proportion relative to the sample as a whole given in bold)

\begin{tabular}{|c|c|}
\hline Category & N (\%) \\
\hline Injury total & $63(17)$ \\
\hline Falls & $22(35)$ \\
\hline Leisure activities & $14(20)$ \\
\hline Muscle and injury strain & $18(29)$ \\
\hline Sports strain & $9(14)$ \\
\hline Personal stress & $53(14)$ \\
\hline Age and age-related conditions total & $45(12)$ \\
\hline Wear and tear/ageing & $43(95)$ \\
\hline Menopause & $2(5)$ \\
\hline $\begin{array}{l}\text { Related to other musculoskeletal diseases } \\
\text { total }\end{array}$ & $40(11)$ \\
\hline Arthritis (unspecified) & $10(31.25)$ \\
\hline PMR relapse & $8(20)$ \\
\hline Osteoarthritis & $7(22)$ \\
\hline Rheumatoid arthritis & $5(16)$ \\
\hline Fibromyalgia & $3(9)$ \\
\hline Others & $7(16)$ \\
\hline Infection total & $39(10)$ \\
\hline Viral & $22(56)$ \\
\hline Unspecified & $10(26)$ \\
\hline Bacterial & $2(5)$ \\
\hline Others & $5(13)$ \\
\hline Occupational strain & $36(9)$ \\
\hline Related to existing chronic diseases total & $24(6)$ \\
\hline Type 2 diabetes & $4(12.5)$ \\
\hline Anaemia & $3(9)$ \\
\hline Thyroid problems & $3(9)$ \\
\hline Weak immune system & $3(9)$ \\
\hline Autoimmune disease & $3(9)$ \\
\hline Others & $9(28)$ \\
\hline Genetic & $21(6)$ \\
\hline Medication total & $18(5)$ \\
\hline Statins & $13(72)$ \\
\hline Medication (general) & $4(22)$ \\
\hline Polypharmacy & $1(5)$ \\
\hline $\begin{array}{l}\text { Consequence/complication of medical } \\
\text { intervention total }\end{array}$ & $16(4)$ \\
\hline Cancer treatment & $7(44)$ \\
\hline Postjoint replacement surgery & $5(31)$ \\
\hline Postsurgical complication & $4(25)$ \\
\hline Environmental total & $14(4)$ \\
\hline Harsh weather & $12(86)$ \\
\hline Bad housing conditions & $2(14)$ \\
\hline $\begin{array}{l}\text { Lifestyle and lifestyle-related conditions } \\
\text { total }\end{array}$ & $10(3)$ \\
\hline Lack of physical activity & $4(40)$ \\
\hline Diet & $3(39)$ \\
\hline Excessive drinking and/or smoking & $2(20)$ \\
\hline Obesity & $1(10)$ \\
\hline
\end{tabular}

terminally ill relative, stressful jobs and dealing with their own serious illness such as cancer or surgery. Thirty-eight per cent (20) of these individuals gave more than one cause. Examples include the following response: "Could be stress of change of lifestyle i.e. 
marriage breakdown, new job, more responsibilities" (58-year-old woman).

With regard to infection, some respondents mentioned a history of travel infection with the inflammation and pain beginning subsequently. Others mentioned that they had an influenza vaccination before the PMR pain started; for example: "...chest infection, got admitted to hospital..." (73-year-old woman); “...flu jab..." (74-year-old man).

Another common cause was occupational strain. Manual occupations were specifically mentioned; including, delivery work, gardening, building and kitchen fitting jobs. Twenty-nine per cent of the respondents gave multiple causes and the most common of these was harsh weather (cold and damp) experienced while doing these jobs. This is not to say that the two were always connected, but $29 \%$ of people from this category mentioned both; as shown in the following examples: "Working in all-weather situations in 50 years as an electrician before health and safety rules" (73-year-old man); “...my work, climbing poles and ladders, leaning out on my belt connecting wires and services" (58-year-old man).

Poor lifestyle choices, including heavy drinking, smoking and poor diet were identified as potential causes: "Manufactured foods. Palm oil. White potatoes. Lack of Vit E. Lack of exercise" (73-year-old woman); "For certain-neglecting my health through bad habits over 15 years-i.e. drinking/smoking too much, not eating regularly..." (67-year-old woman).

Statins were the commonest group of drugs to which respondents attributed PMR, accounting for $72 \%$ (13) of those that attributed their PMR to medication: "After my heart attack on 28th Dec 2013 my prescription for Atorvastatin was increased from $20 \mathrm{mg}$ to $80 \mathrm{mg}$. 7 weeks later my PMR symptoms started. I was taken off Atorvastatin and later back on. My PMR became worse" (84-year-old woman).

Respondents also said their PMR resulted from medical intervention, in particular surgery, with some highlighting a knee or hip replacement that had put extra strain on their shoulders or the other leg, which they felt contributed to their PMR.

Genetic causes were mentioned by $9 \%$ of the cohort. The mean age of this group was 65.8 years and $75 \%$ were female. This factor was largely given as a single cause; except for $15 \%$ of the respondents who coupled genetic causes with occupational strain, personal stress and a viral infection.

\section{DISCUSSION}

This study has identified a wide variety of factors that respondents believe caused their PMR. Commonest proposed causes were injury, personal stress and the ageing process. While some respondents cited a single cause; others suggested multiple factors in the development of their condition. However, the majority of those in this cohort did not know what had caused their PMR. This study is the first to examine patient beliefs about the causes of PMR.
One strength of this study is the analytical framework used. Using a summative content analysis approach was advantageous because it is an unobtrusive and nonreactive way to study opinions. ${ }^{10}$ Additionally, independent coding by two researchers, who were able to achieve a high level of agreement in their coding of the data and subsequent detailed discussions, improves the credibility of the results. A further strength is the large sample size which was composed providing a representative sample of the primary care PMR population. Furthermore, respondents in this study had been very recently diagnosed with PMR prior to questionnaire completion, minimising recall bias. A limitation of the study, however, is that an analysis of short written responses does not allow for consideration of broader contextual factors related to patients' beliefs. For instance, patients were not asked about the information sources they had drawn on in forming these causal beliefs. We therefore do not know how many patients had actively investigated causation themselves, either online or in discussions with treating clinicians, or whether patients were basing these beliefs solely on experiential and/or anecdotal evidence. Future research could therefore build on the breadth of views accessed in this study through an in-depth qualitative investigation of people's perceptions, allowing potential causal explanations and their underpinnings to be explored in greater detail.

A varied range of causal beliefs have also been observed in relation to patients with arthritis ${ }^{11}$ and similar studies of patients with arthritis have shown that opinions about causation influence the psychological aspect of the disease, which may in turn affect coping. ${ }^{8}$ This may also be the case with PMR because of the risk of serious complications such as giant cell arteritis, which untreated can lead to blindness. ${ }^{12}$ A study on Latina women with arthritis stressed the need for having emotional and social support to deal with these psychological aspects of rheumatological disease,${ }^{13}$ and as such understanding illness beliefs may help improve coping for patients with PMR.

A study of the impact of illness beliefs in arthritis (of various forms, including osteoarthritis and RA) on patients in the Dominican Republic showed that patients believed that their arthritis was caused either by contact with water or that it was a test from God. As a result, patients avoided contact with water and prayed as a way of coping. Although these views are unconventional, the study showed that having these passive explanatory models ${ }^{14}$ help patients accept their illnesses.

A sizable proportion of respondents in this study said that their PMR was caused by the normal ageing process. This tendency to normalise illness has also been shown to be common in patients with arthritis; ${ }^{15}{ }^{16}$ Sanders et al, ${ }^{15}$ for instance, reported that their interview participants, aged 51-91 years, 'referred repeatedly to a natural degenerative theory of causation' for their arthritis. While such an explanation can help in the acceptance of the illness, it may also lead to a delay in seeking care. Other common causes cited, such as stress and injury, show similarity with 
the findings of Berenbaum et al who explored patient beliefs in France about RA and SpA through in-depth interviews. In relation to RA, they found that personal stress, often resulting from a distressing life event, was frequently cited as a trigger for onset of the condition, as was intense physical activity, which may be comparable to the category of 'injury' identified in the present study, found here to include 'muscle and injury strain' and 'sports strain'. The similarities with Berenbaum et $a l \mathrm{~s}^{8}$ findings suggest that causal beliefs about PMR are unlikely to be entirely unique to the condition, and may instead share similarities with beliefs relating to other rheumatic conditions, particularly RA, perhaps reflecting the inflammatory nature of both conditions.

While citing the natural ageing process as a cause positions the onset of PMR as somewhat inevitable and therefore out of the patient's control, beliefs about personal stress and injury suggest external factors-both psychological and physical—that patients may have some control over; perhaps suggesting a degree of agency on their part. The degree of agency patients perceived themselves as having over their PMR could have implications for how they view their ability to self-manage the condition; therefore, understanding these beliefs can also have implications for the clinical advice given to patients about self-management of PMR.

Some of the causes of PMR that were cited are medically unlikely; for example, diet and insect bites. While it can be unhelpful if patient beliefs are at odds with clinical knowledge, understanding these beliefs enables them to be addressed and may help patients make sense of their condition. Therefore, even if patients' beliefs are unlikely to reflect reality, they can be important for how patients cope with their condition, and therefore these beliefs are no less valid. For example, in the case of the patients in the Dominican Republic, although their belief that water caused their arthritis was not correct, it was because of this belief that they attached great importance to draining knee effusions, and this lead to care seeking and improved disease management. ${ }^{14}$

Finally, a study by Lambert et $a{ }^{17}{ }^{17}$ regarding patient and physician views on arthritis suggested that exploring patient beliefs about disease causation can help in identifying and addressing unhelpful beliefs. For example, if a patient expects that the medication will eliminate pain and reverse joint deformity, when this outcome is not achieved patients may struggle to trust their physicians. ${ }^{17}$ Exploring patients' beliefs of causation, prognosis and treatment helps in allaying fears, patient education and treatment adherence and can result in more realistic patient expectations.

The findings presented in this study suggest a need for greater dialogue between patients and clinicians, so that clinicians explore such thoughts/ideas in order to dispel any unhelpful illness beliefs. ${ }^{8}$ For example, if a patient harbours the belief that exercise may have caused their PMR, this may deter them from even doing the light joint exercises that are recommended as part of PMR management. While addressing such beliefs should already be part of routine clinical practice, making clinicians more aware of the most common causal beliefs held by patients with PMR may place them in a stronger position to identify and address unhelpful patient beliefs, particularly in cases where patients are not forthcoming in expressing these beliefs in the consultation. The findings presented here can therefore provide clinicians with a basis from which to explore these beliefs in relation to individual patients.

This study provides interesting patient insights into the cause of their PMR and may prompt additional hypotheses that warrant further study. Some patient attributions including infection and age have been postulated previously, ${ }^{2-4}$ although no definite evidence exists at present. Likewise no clear evidence exists at present regarding an association between either injury or stress and PMR onset; however, though not conclusive, some studies have found an association between stress and increased disease activity in certain rheumatic conditions, ${ }^{18}$ and it may therefore be useful to explore this association in future research specifically in relation to PMR. Some patients attributed their PMR to medications, especially statins. Myalgia is a commonly reported side-effect of statins, and this finding lends support to evidence from de Jong et $a l^{19}$ who found an association between statin use and the occurrence of PMR. However, to date there is no evidence of a causal relationship between statins and PMR, and therefore further research exploring the nature of this association would be beneficial.

Thus, to date, although the cause of PMR is not known, patients with PMR reported a range of factors which they believe to have caused their illness. Commonly cited causes included injury, personal stress, ageing, medication and chronic illness. Engaging in dialogue with patients to understand their illness beliefs may help patients to make sense of their illness and influence coping and self-management strategies and thus have a positive impact on the clinical management of PMR.

Acknowledgements The authors would like to thank the study participants, as well as the wider PMR Cohort Study team.

Contributors MT analysed all of the data and wrote the manuscript. BS contributed to data analysis and to the writing of the manuscript. SM conceptualised and developed the idea for the study as part of the broader research programme, contributed to data analysis and to the writing of the manuscript. CDM developed the idea for the study, contributed to data analysis and to the writing of the manuscript. SLH developed the idea for the study, contributed to data analysis and to the writing of the manuscript. All authors critically edited the manuscript and read and approved the final version.

Funding This work was supported by an Arthritis Research UK Clinician Scientist Award received by CDM who funded the PMR Cohort Study (grant number 19634). MT was funded to carry out the present research through an INSPIRE summer studentship. CDM is funded by the National Institute for Health Research (NIHR) Collaborations for Leadership in Applied Health Research and Care West Midlands, the NIHR School for Primary Care Research and a NIHR Research Professorship in General Practice (NIHR-RP-2014-04-026).

Disclaimer The views expressed are those of the author(s) and not necessarily those of the NHS, the NIHR or the Department of Health. 
Competing interests None declared.

Ethics approval Ethical approval for the study was received from the Staffordshire Local Research Ethics Committee (REC reference number: 12/WM/0021).

Provenance and peer review Not commissioned; externally peer reviewed.

Data sharing statement No additional data are available.

Open Access This is an Open Access article distributed in accordance with the Creative Commons Attribution Non Commercial (CC BY-NC 4.0) license, which permits others to distribute, remix, adapt, build upon this work noncommercially, and license their derivative works on different terms, provided the original work is properly cited and the use is non-commercial. See: http:// creativecommons.org/licenses/by-nc/4.0/

\section{REFERENCES}

1. Mackie SL. Polymyalgia rheumatica: pathogenesis and management. Clin Med (Lond) 2013;13:398-400.

2. Kermani TA, Warrington KJ. Polymyalgia rheumatica. Lancet 2013;381:63-72.

3. Pipitone N, Salvarani C. Update on polymyalgia rheumatica. Eur J Intern Med 2013;24:583-9.

4. Subrahmanyam P, Dasgupta B. Polymyalgia rheumatica. Medicine 2006;34:427-30.

5. Salvarani C, Cantini F, Boiardi L, et al. Polymyalgia rheumatica. Best Pract Res Clin Rheumatol 2004;18:705-22.

6. Soriano A, Landolfi R, Manna R. Polymyalgia rheumatica in 2011. Best Pract Res Clin Rheumatol 2012;26:91-104.

7. Srinivasan TN, Thara R. Beliefs about causation of schizophrenia: Do Indian families believe in supernatural causes? Soc Psychiatry Psychiatr Epidemol 2001;36:134-40.
8. Berenbaum F, Chauvin P, Hudry C, et al. Fears and beliefs in rheumatoid arthritis and spondyloarthritis: a qualitative study. PLOS ONE 2014;9:e114350.

9. Muller S, Hider SL, Helliwell T, et al. Characterising those with incident polymyalgia rheumatica in primary care: results from the PMR Cohort Study. Arthritis Res Ther 2016;18:200.

10. Hsieh FH, Shannon SE. Three approaches to qualitative content analysis. Qual Health Res 2005;15:1277-88.

11. Kay EA, Punchak SS. Patient understanding of the causes and medical treatment of rheumatoid arthritis. $\mathrm{Br} J$ Rheumatol 1988;27:396-8

12. Mackie LS, Mallen CD. Polymyalgia rheumatica; clinical review. BMJ 2013:347:f6937

13. Abraído-Lanza AF, Revenson TA. Coping and social support resources among Latinas with arthritis. Arthritis Rheumatol 1996;9:501-8.

14. Niu NN, Davis AM, Bogart LM, et al. Patient disease perceptions and coping strategies for arthritis in a developing nation: a qualitative study. BMC Musculoskelet Disord 2011;12:228.

15. Sanders C, Donovan J, Dieppe P. The significance and consequences of having painful and disabled joints in older age: co-existing accounts of normal and disrupted biographies. Sociol Health IIIn 2002;24:227-53.

16. Sanderson T, Calnan M, Morris M, et al. Shifting normalities: interactions of changing conceptions of a normal life and the normalisation of symptoms in rheumatoid arthritis. Sociol Health IIIn 2011;33:618-33.

17. Lambert BL, Butin DN, Moran D, et al. Arthritis care: comparison of physicians' and patients' views. Semin Arthritis Rheum 2000;30:100-10.

18. Hassett AL, Clauw D. The role of stress in rheumatic diseases. Arthritis Res Ther 2010;12:1-2.

19. De Jong HJ, Saldi SR, Klungel OH, et al. Statin-associated polymyalgia rheumatica. an analysis using WHO global individual case safety database: a case/non case approach. PLOS ONE 2012;7:e41289. 